\title{
An Underwater Robotic Network for Monitoring Nuclear Waste Storage Pools
}

\author{
Sarfraz Nawaz ${ }^{1}$, Muzammil Hussain ${ }^{1}$, Simon Watson ${ }^{2}$, Niki Trigoni ${ }^{1}$, and Peter \\ N. Green ${ }^{2}$ \\ 1 Oxford University Computing Laboratory \\ Wolfson Building, Parks Road, \\ Oxford OX1 3QD \\ \{Sarfraz.Nawaz, Muzammil.Hussain, Niki.Trigoni\}@comlab.ox.ac.uk \\ 2 School of Electrical and Electronic Engineering \\ University of Manchester \\ Sackville Street Building \\ Manchester M60 1QD \\ simon.watson@postgrad.manchester.ac.uk \\ peter.n.green@manchester.ac.uk
}

\begin{abstract}
Nuclear power provides a significant portion of our current energy demand and is likely to become more wide spread with growing world population. However, the radioactive waste generated in these power plants must be stored for around 60 years in underwater storage pools before permanent disposal. These underwater storage environments must be carefully monitored and controlled to avoid an environmental catastrophe. In this paper, we present an underwater mobile sensor network that is being developed to monitor these waste storage pools. This sensing system will also be used in very old storage pools to build maps of their internal structure which can then be used for waste removal and pool decommissioning. In this paper, we outline the unique challenges of our application scenario which include robot localization in cluttered underwater environments and the effect of location errors on environment mapping. We also list other industrial applications that can benefit from our underwater sensor network.
\end{abstract}

\section{Introduction}

According to the World Nuclear Association [1], there are a total of 437 operational nuclear reactors around the world supplying approximately $15 \%$ of the total electricity consumption. In the UK alone, there are 19 nuclear power plants generating electricity for civilian use and 25 old power plants that are in various stages of shutdown and decommissioning. As the world population grows, increased energy demand is likely to make nuclear energy generation more wide spread. However, the biggest issue associated with nuclear power is the generation of radioactive waste which must be managed and stored over a long period of time. This radioactive waste can be classified as low level waste (LLW), intermediate level waste (ILW) and high level waste (HLW). Low level waste con- 
tains paper, tools, clothing and other material that produce very small amount of radioactivity. It is generally incinerated or processed as ordinary waste. Intermediate level waste comprises of chemicals, resins and metal fuel cladding. It contains higher amounts of radioactivity and thus requires shielding. It is sometimes stored in short term storage facilities for a few weeks or months before being solidified and buried in near surface trenches. Spent nuclear fuel and products of the fission reaction form high level waste. It generates the highest amount of radioactivity and thus requires shielding and careful handling. It is held in interim storage for 20 to 60 years before it can be transferred to permanent disposal sites. Some of these high level wastes can have a half-life ranging from hundreds of thousands to millions of years and thus require special treatment. The most popular proposal is deep geological disposal in which the waste would be solidified in glass or ceramic through a process called vitrification and then buried in very deep rock formations ranging from $300 \mathrm{~m}$ to $800 \mathrm{~m}$ below the earth surface [2]. There are also some proposals to bury these wastes under the seabed [3].

Although, deep geological disposal is in its advanced stages of research, there are no fully functional waste disposal repositories available at present. Even if such repositories were available, it is still necessary to store this waste in cooling ponds for 20 to 60 years to remove the heat that is continuously generated by this waste. After this extended underwater storage, the amount of heat generated is reduced to a level that is suitable for deep rock burial. During this extended underwater storage, the cooling ponds must be carefully monitored for temperature hot-spots and leakage in storage canisters. We are developing an underwater mobile sensor network for these nuclear waste storage pools in collaboration with our partners at University of Manchester and the National Nuclear Laboratory. This network will consist of a swarm of small scale robots. Each robot will be approximately $10 \mathrm{~cm}$ in diameter and will collaborate with other robots to monitor the conditions inside the storage pool. The cluttered underwater environment of these pools presents significant difficulties for accurate robot localization. Inaccurate robot positions in turn influence the higher layer tasks of environment mapping and high density spatial sampling. In this paper, we discuss these challenges in detail and highlight these issues with our preliminary results. We also list underwater processes in other industrial applications outside the nuclear domain that can benefit from our mobile sensing system.

The rest of this paper is organized as follows. Section (2) introduces the overall architecture and design of our mobile sensing system. Section (3) outlines the issues faced by robot localization algorithms and some preliminary experiences. Section (4) discusses the challenges in performing mapping and exploration tasks. Section (5) outlines the open issues and a research vision that we intend to pursue. Section (6) presents the simulation environment that has been set up to test and analyze the performance of various algorithms. Section (7) lists other industrial applications of our mobile sensor network. Section (8) reviews related work and Section (9) concludes this paper. 


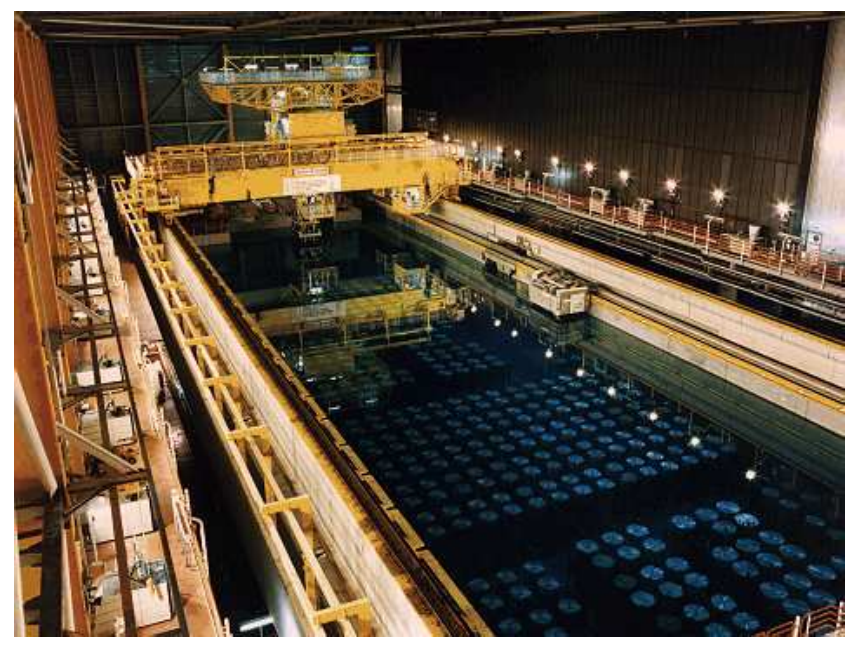

Fig. 1: Modern Storage Pool

\section{System Architecture}

In this section, we describe our application environment in detail and outline a high level architecture of our mobile sensing system. There are two different types of storage pools that we are targeting. In the following, we describe the differences between these two types and then present our system design.

The first type of storage ponds are modern, well maintained and clean pools. These are generally indoor concrete structures that are designed to be resistant to movements generated by events like earthquakes. Their sizes vary with the largest ones approaching the dimensions of an Olympic sized swimming pool i.e. $50 \mathrm{~m} \times 25 \mathrm{~m}$. These pools can be as deep as $20 \mathrm{~m}$ and are equipped with pumps and heat exchangers. The waste is sealed in steel flasks and then put in large skips. These skips are stacked on top of each other with the help of an overhead crane assembly. This creates an underwater landscape of rows of towers of skips. The distance between the surface of water and the top of skip towers is generally one to two meters. This thick layer of water prevents any radiation from escaping the pool. Pools are also equipped with cleaning systems to control water quality that is necessary to prevent any corrosion of storage skips. Fig. (1) shows a photograph of one such storage pool at a reprocessing facility in the UK. In these ponds, our mobile sensor network will be used to perform dense spatial sampling of temperature, $\mathrm{pH}$ and radioactivity. It will also be used to monitor for any leakages. Our specific research objective is to accurately sample temperature and radioactivity fields in these storage ponds using a swarm of resource constrained robots.

The second type of storage ponds are very old ponds that were built during the early 1950s and used until late 1980s. Over the years, a lot of intermediate and high level waste has been dumped in these ponds. The metal structures 


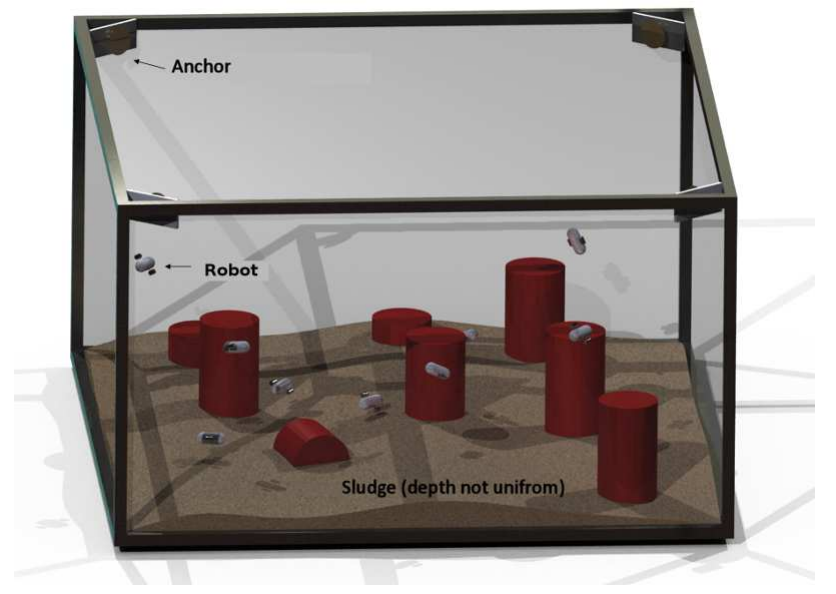

Fig. 2: Underwater Sensor Network Architecture

and spent fuel rods in these ponds have decomposed and formed a thick sludge that has settled on the bottom over time. This sludge may contain pockets of hydrogen and other solid objects. Visibility inside these ponds is very poor because of the suspended particulates. Skip towers have also toppled over and the internal structure is not known. There are no detailed records regarding the exact contents of these ponds. Some of these ponds are extremely hazardous and only very little human activity is allowed in the vicinity of these ponds. It is necessary to gain more information about the internal structure of these ponds so that the waste can be removed and stored in modern well maintained ponds until deep geological disposal is available. In these ponds, our specific research objective is to build accurate maps of the internal structure of the ponds using a swarm of resource constrained robots. Both tasks of spatial sampling (in new ponds) and mapping (in old ponds) require robots to be able to localize themselves within the pond. The main questions discussed in this paper are, how the presence of clutter - stored nuclear waste - impacts localization errors, and how these errors influence the spatial sampling and mapping processes.

Fig. (2) shows an overall architecture of our underwater mobile sensing system. The storage pond is instrumented with a number of fixed anchor nodes. These anchor nodes periodically transmit acoustic beacons that are used by mobile nodes to determine their position inside the pool. These nodes also act as collection sinks and are used to receive data transmitted by robots on the acoustic channel. Robots can also communicate with each other and perform distance measurements among themselves using acoustic signals. This allows the robots to collaborate with each other during the localization, sensing and mapping tasks. Both the payload and propulsion system of the underwater robots are currently under investigation. At this point, it is envisaged that the the payload will initially consist of a pressure sensor to measure depth, a temperature sensor and a 


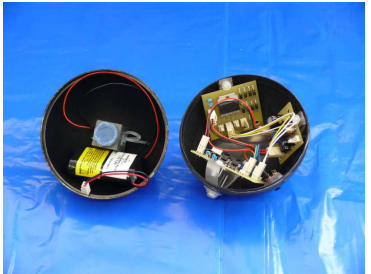

(a) Robot Internals

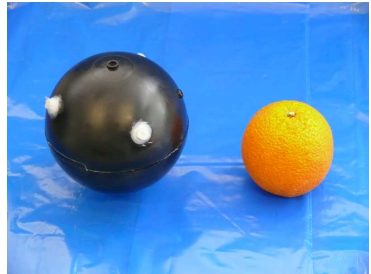

(b) Robot size

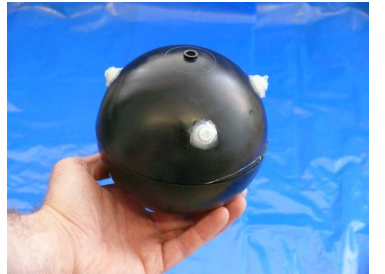

(c) Prototype Robot

Fig. 3: Size comparison of prototype robot

number of obstacle detection sensors that will be based on the communication and positioning acoustic transducers. There will also be a hardness sensor based on the same technology and an optical based turbidity sensor. The propulsion system is split into the $\mathrm{z}$ plane and $\mathrm{x}-\mathrm{y}$ plane and will provide at least 4 degrees of freedom (surge, sway, heave and yaw) [4]. Movement in the z-plane will come from propellers while the $\mathrm{x}-\mathrm{y}$ plane movement will be achieved by a combination of propellers and/or miniaturized vortex ring thrusters [5]. The propulsion systems will be controlled by an embedded system in the form of a 32-bit microprocessor with DSP. Each robot is expected to be approximately $10 \mathrm{~cm}$ in diameter. Fig. (3) shows an initial prototype version of our robot.

Fig. (4) shows how different components of the software system interact with each other and the hardware components. The lowest layer consists of various hardware components that include acoustic communication and ranging, various sensors and propulsion system. These hardware components are in various stages of development. Middle level layers are responsible for localizing the robot and for performing reactive obstacle avoidance. These middle layer components have been implemented in a simulation environment. The higher layer consists of our two distinct applications i.e. spatial sampling in new ponds and exploration and mapping in old ponds.

\section{Robot Localization}

\subsection{Background}

Location awareness is a basic requirement for a robot swarm to explore and map an enclosed underwater environment such as a nuclear waste storage pond. It is necessary for the robots to have accurate position information not just for actual mapping but also for navigating through the pond. Anchor nodes, nodes connected to external reference systems that supply positional information, enable the use of trilateration for localizing robots. The technique exploits geometrical constraints, given the locations of a number of anchors and distances to them, to calculate the position. Even though for three-dimensional positioning we require 


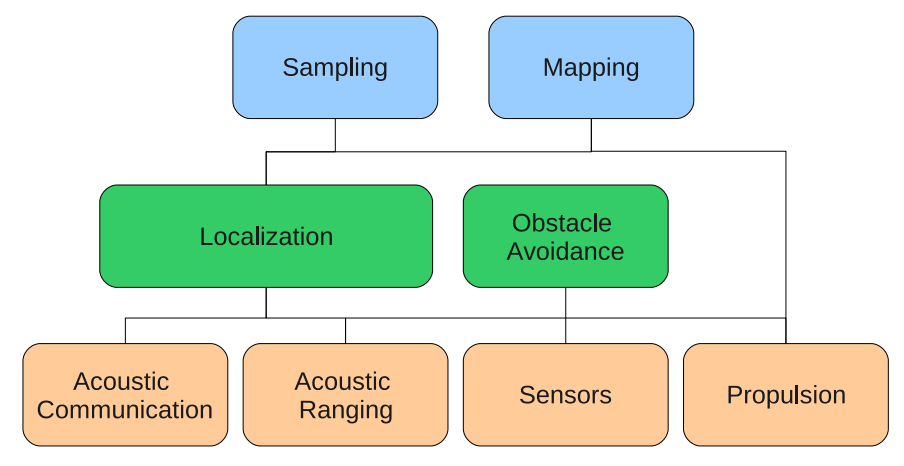

Fig. 4: Software Architecture

at least four anchors, the underwater robots we are designing have pressure sensors which can be used to determine the depth (z-coordinate) of the robot. This obviates the need for a fourth anchor.

Distance measurements to the anchors, in noisy environments, have small Gaussian errors [6], thus introducing an error in the calculated position. In large networks, where the number of anchors is small compared to the remaining nonlocalized nodes, it could be the case due to the limited transmission range of the acoustic communication and the attenuating effects of cluttered environments, that all anchors would not be heard by all the nodes in the network. It is possible that a particular unpositioned node cannot hear the required number of anchors in order to calculate its position thus requiring neighbouring nodes which have already been localized (owing to their proximity to the required number of anchors) to assist them. These neighbouring nodes are used as virtual anchors and the process is called iterative localization. However these virtual anchors, having previously localized themselves, contain inherent errors in their own positions. These errors, compounded by the noisy distance measurements, could propagate via successive iterative localization steps. It is vital to control the propagation of error due to noisy distance measurements and inaccurate virtual anchors positions.

Iterative localization has been a well researched topic for the past few years. Collaborative multi-lateration is introduced in [7] where location information from across multiple hops is used to localize a node. In [8], the authors propose a method to quantify the errors introduced by virtual anchors and noisy range measurements. Each node can thus prevent the accumulation of localization errors by maintaining a registry of its neighbouring anchor nodes and using those anchors in trilateration that have the smallest errors. Another approach [9] to quantifying error in iterative localization takes into account the geometric relationships between the anchors during selection. Localization in underwater environments has also been an active area of research [10]. However, almost all of this work deals with localizing sensor nodes in open sea environments $[11,12]$. 
Our research, on the other hand, deals with localizing sensing robots in cluttered underwater environments. This is discussed in detail in the following subsection.

\subsection{Localization in Cluttered Underwater Environments}

One of the primary areas of interest in this project is the challenges faced by a network of robots in exploring cluttered environments. Clutter is generally irregularly shaped obstacles that obstruct acoustic propagation paths between robots or worse introduce multipath acoustic communication.

The underlying concept behind range-based localization is that the distance between the robot and the anchors can be obtained by sending a communication signal between them and the distance is derived from the characteristics of the received signal. In case of acoustic/ultrasound signals, the propagation time translates into the distance travelled, assuming that we know the velocity of sound in that particular medium accurately. This, however, hinges on the assumption that the signal travels straight from the anchor to the non-localized robot along the shortest path, without bouncing off any obstacles in between. Such signals are called Line Of Sight (LOS) signals. The measurements from such signals would yield the least distance errors. However in reality, in cluttered environments with obstacles between the transmitter and receiver, it is possible that a large number of signals are Non Line Of Sight (NLOS). Such signals have large distance measurement errors.

There has been extensive research in the mitigation of non line-of-sight measurements, particularly in cellular network research. It was motivated by the requirement put forth by Federal Communications Commission (FCC) to cellular operators to be able to locate a mobile handset within an accuracy of 300 meters for $95 \%$ of calls [13]. In current literature $[14,15,16,17,18,19,20,21]$ there are three general methods to deal with NLOS readings. The first method attempts to identify and use only LOS measurements. Distinguishing NLOS from LOS distance measurements could either be done using a time-varying hypothesis test [14], a probabilistic model [18] or residual information [15, 21]. The second method incorporates both LOS and NLOS distance measurements with appropriate weighting to minimize the contribution of NLOS observations. Here the primary assumption made is that the number of LOS readings is much greater than the number of NLOS readings $[16,17]$, which may not be the case in our underwater cluttered environment. The third method advocates the use of scattering models. This method takes into account the propagation characteristics of the channel and then directly determines the actual line-of-sight distance using the NLOS readings using a scattering model $[19,20]$. However this requires perfect knowledge of the underwater environment, including the topology and type of clutter, which may not be possible in most cases.

We have simulated robot localization in the presence of LOS signals and NLOS signals in Fig. (5). In Fig. (5a), we see that the smallest localization error is obtained when only LOS distance measurements are used. In Fig. (5b) we see that localization error increases with a smaller number of LOS distance measurements, even though we have filtered out NLOS measurements. Fig. (5c) 


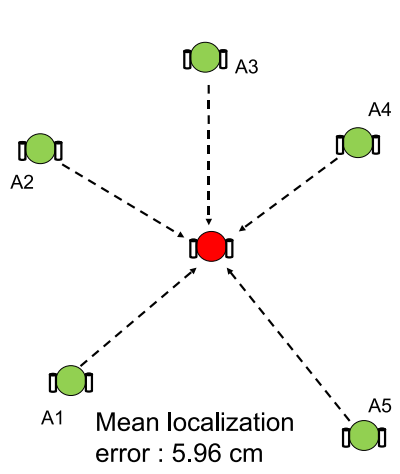

(a) LOS anchors only

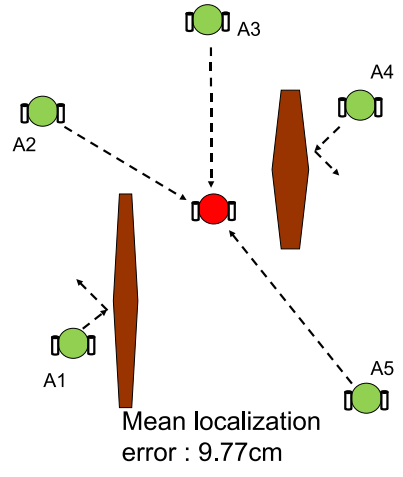

(b) LOS and NLOS anchors

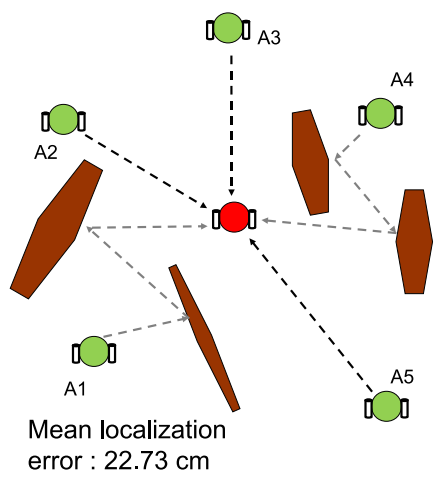

(c) NLOS anchors filtered

Fig. 5: Robot localization errors when LOS distance estimates have a small Gaussian error $(\sigma=6.5 \mathrm{~cm})[6]$ and NLOS distance estimates have a large constant error $(30 \mathrm{~cm})$

shows that using NLOS measurements in addition to LOS measurements gives a large localization error. Hence we can conclude that not only should we filter out NLOS measurements but also have enough LOS measurements to get an accurate position estimate.

From the preliminary experiments we have conducted, we envisage two scenarios as illustrated in Fig. (6). In the first scenario, the clutter is impermeable to acoustic signals. Here only reflected non line-of-sight (NLOS) signals, which have large errors in distance estimates, are present. These have to be detected and filtered from the remaining range measurements before calculating the position. In the second case, the clutter is actually permeable to acoustic signals whereby the signal can 'resonate' through the obstacle without losing all the energy. In this case, the distance estimate of the NLOS signal would be comparable to that of a LOS signal. 


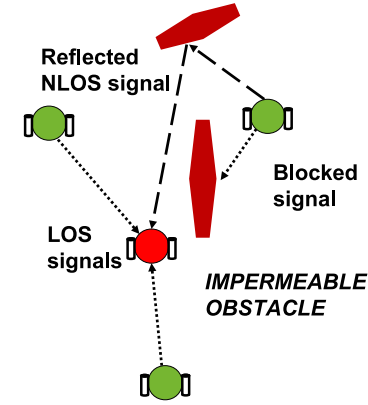

(a) Non-Permeable Obstacles

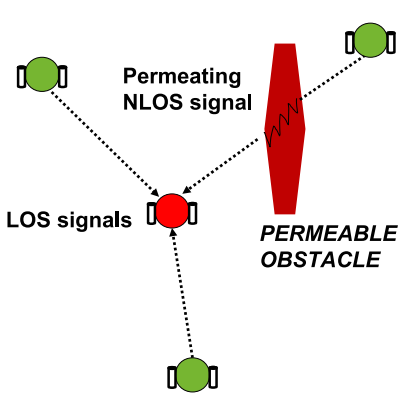

(b) Permeable Obstacles

Fig. 6: Possible types of obstacles in a nuclear storage tank. Permeable obstacles giving rise to 'near-LOS' NLOS range measurements

When the majority of the NLOS measurements are of the first type i.e., reflected NLOS, it may be the case that filtering out NLOS measurements (anchors) could lead to a non-localized robot not having access to the required number of anchors. For example, in Fig. (5c), if three of the five measurements had been NLOS, the non-localized robot would not have been able to calculate its position. Also the reduced number of LOS anchors increases the error in the position estimate. In this scenario it may become necessary to develop a strategy to deploy previously localized robots to help their non-localized neighbours to localize, so as to maximize the chances that a node, at any given position in the underwater pond, can localize itself accurately. At first we would want to work with the static case where the robots are stationary. Here emphasis is put on the initial position of the robots so as to enable all/most of the robots to be localized.

Now that we have introduced the challenges of robot localization in cluttered underwater environments, in the next section, we discuss how these localization errors effect the mapping task.

\section{Exploration and Mapping}

Creating maps of the bottom of old waste storage ponds where sludge and other particulate material has settled down over long periods of time is an important aspect of our mobile sensing system. In order to create these maps, the robots must be aware of their surroundings as well so that they can safely move around in the pond without colliding with the waste storage canisters. Thus, to accomplish this task, the robots must explore and map the entire pond. These robots must also collaborate with each other to map the environment efficiently. In this section, we discuss some related work on robotic mapping and exploration, and outline the challenges of performing the mapping task in the pesence of 
robot localization errors. We also present some preliminary simulation results that highlight these issues.

When a robot is placed in an unknown environment and it does not have access to any location information, it has to determine its location and build the map of the environment at the same time. This problem is known as Simultaneous Localization and Mapping (SLAM) or Concurrent Mapping and Localization $(\mathrm{CML})$ and has received considerable attention from the robotics research community. Smith and Cheeseman [22] used an Extended Kalman Filter (EKF) to solve the SLAM problem for the first time and since then it has been a highly active field of research. Thrun [23] provides an extensive survey of SLAM techniques.

When the location information is available to a robot, the problem of mapping the environment becomes easier as compared to SLAM. However, noisy sensor measurements and complex environments can still pose significant challenges to mapping and exploration algorithms. The most widely used family of algorithms used to map environments with known robot locations is called Occupancy Grid Mapping. Occupancy grid based mapping was introduced by Elfes [24] and later a Bayesian statistical basis of this approach was developed by Moravec [25]. Moravec [26] also extended this approach to build three-dimensional maps using stereo cameras. Occupancy grids have also received a wider acceptance in robotics because they are commonly used as input to algorithms for path planning, collision avoidance, sensor fusion etc. In our system, a group of anchor nodes provide an infrastructure that is used by robots to determine their positions. Therefore, we use occupancy grids for building the maps of underwater environments of the storage pools.

The basic idea of occupancy grid mapping is that the space that has to be mapped is divided in small uniform sized cells, for example, $10 \mathrm{~cm} \times 10 \mathrm{~cm}$. For each cell, the robot maintains a probabilistic belief about the occupancy of the cell. In the beginning, no information is available and the status of each cell is unknown. As the robot moves around, it gathers measurements from its perception sensors (e.g. sonar, laser range finder, stereo cameras or some other sensor) and the corresponding cells are updated with these measurements according to a Bayesian reasoning approach. In order to determine which cells to update, the robot must know its position in the environment and the characteristics of its sensors. For example, if a robot is using a sonar sensor to perceive its environment, then all the cells $m_{i}$ of the occupancy grid that lie within the beamwidth of sonar sensor are updated according to the Bayes rule using the log odds representation as,

$$
l_{t, i}=l_{t-1, i}+\log \frac{p\left(m_{i} \mid x_{t}, z_{t}\right)}{1-p\left(m_{i} \mid x_{t}, z_{t}\right)}
$$

where $x_{t}$ is the location of the robot at time $t, z_{t}$ is the sonar measurement and $p$ is the occupancy probability derived from a sensor model given the range measurement $z_{t}$ returned from the sonar and $l_{t, i}$ is 


$$
l_{t, i}=\log \frac{p\left(m_{i} \mid x_{1: t}, z_{1: t}\right)}{1-p\left(m_{i} \mid x_{1: t}, z_{1: t}\right)}
$$

Generally, the $\log$ odds of prior probability $l_{0}$ is set to zero for all cells $m_{i}$. The $\log$ odds representation provides an easy update rule in the form of Eq. (1). When it is required to determine whether a cell $m_{i}$ is occupied or free, the probability $p\left(m_{i} \mid x_{1: t}, z_{1: t}\right)$ can be recovered as

$$
p\left(m_{i} \mid x_{1: t}, z_{1: t}\right)=1-\frac{1}{1+\exp \left(l_{t, i}\right)}
$$

and compared against thresholds thres-occ and thres-free to decide on its occupancy,

$$
p\left(m_{i} \mid x_{1: t}, z_{1: t}\right)\left\{\begin{array}{l}
\geq \text { thres-occ } \quad m_{i} \text { is occupied } \\
\leq \text { thres-free } \quad m_{i} \text { is free } \\
\text { otherwise } m_{i} \text { is unknown }
\end{array}\right.
$$

The probabilistic belief for each cell in the grid is derived using sensor measurements and robot location. Therefore, any measurement or location error affects the occupancy grid. We have shown in Section (3) that the cluttered environment of the storage pools can introduce significant errors in estimated robot positions. These location errors in turn introduce anomalies in the storage pool maps built by robots in the form of occupancy grids. Fig. (7a) shows a $6 \mathrm{~m}$ $\times 6 \mathrm{~m}$ simulated pool where two towers of storage skips have been placed. Anchor nodes have been placed at four out of the eight corners of pool and a single robot moves through this lightly cluttered pool localizing itself through singlehop trilateration. For direct line of sight distance estimates, a small error from a normal distribution $N(0, \sigma)$ with $\sigma=5 \mathrm{~cm}$ is introduced. For non line of sight distances between the anchors and robots, a uniform distribution $U(0, b)$ with $b=30 \mathrm{~cm}$ is used to introduce errors. The robot uses sonar measurements and its estimated location to build a 2D map of the simulated pool using frontier based exploration and mapping [27]. A number of such 2D maps can be built at various depths to create a three dimensional map of the environment. Further details of our simulation environment are described in Section (6). Fig. (7b) shows a 2D map that is built using the perfect location information without any errors. Fig. (7c), on the other hand, shows a map when the estimated robot location contains significant errors due to non line of sight (NLOS) distance estimates between the robot and the anchor nodes. These location errors not only distort the map but since the partial map is also used by the robot for exploration and path planning, they also affect the efficiency of the mapping process. Our aim is to quantify the effect of these errors on the mapping process and then develop efficient exploration strategies that can mitigate the effect of these errors on the mapping process. 


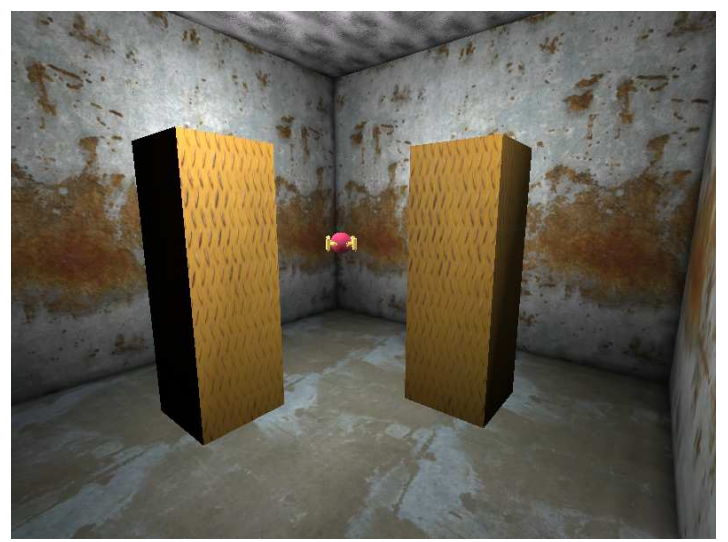

(a) Simulated Cluttered Pond

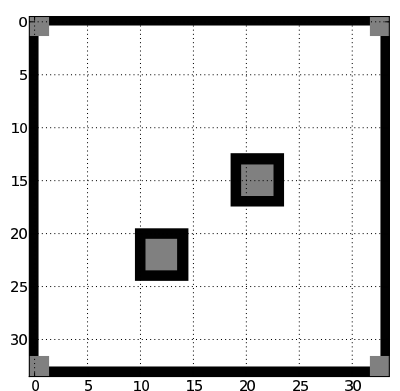

(b) Mapping without location errors

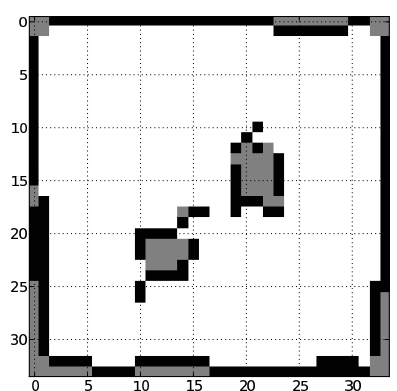

(c) Mapping with location errors

Fig. 7: Mapping in cluttered ponds

\section{Open Research Issues}

Now that we have described the specific challenges of robot localization and its effects on mapping and exploration tasks in the previous two sections, we now present a vision that we intend to pursue in our future research. We envisage three different types of environment mapping systems using our swarm of robots. In the following, we outline these three systems and the individual questions that we intend to answer for each of these systems.

Our first system consists of robots that have been classified in two different categories depending on their role. We term the robots belonging to the first category as localizers and those belonging to the second category as explorers. The role of the localizer robots is to position themselves in such a manner that the explorer robots can localize themselves and thus explore the cluttered environment. The main challenge of this approach is to optimally place localizers so that the explorers can build an accurate map of the environment. 
In our first system, there is no feedback mechanism between the localization and the exploration tasks. Explorers only explore those regions where the localizers are available to provide positioning infrastructure. In the second system that we envisage an explicit feedback mechanism is available between the explorers and localizers. Thus the explorers can request the localizers to move to a certain region to provide positioning infrastructure so that that region can be explored and mapped. The main challenge of this approach is to design efficient resource allocation strategies that can be used to assign localizers to explorers.

In our third system, there is no distinction of roles and each robot performs both of the tasks simultaneously i.e. each robot not only explores and maps the environment but it also helps its neighboring robots to localize themselves. Thus in this final system, these tasks are very tightly coupled. The main challenge of this approach is to design an efficient coordination strategy among the robots that maximizes the accuracy of the built map.

The specific question that we would like to answer is which one of these three systems can perform environment mapping more accurately given a fixed number of robots, limited energy and the harsh cluttered environment.

\section{Simulation Environment}

The target scenario of our research project is cluttered underwater environments. This is a unique application scenario that has not been extensively addressed in research literature. Although, there is a large body of work on underwater robots, most of the readily available robot simulators do not provide any means of simulating underwater environments. Stage is probably the most widely used simulator in robotic research community. It provides a $2 \mathrm{D}$ environment in which virtual robots can be spawned and controlled by clients. It is generally used with Player [28] which provides a set of standard interfaces. Stage uses very simple and computationally efficient models for the virtual robots and sensors. The advantage of this approach is that the simulation can be scaled to a very large number of robots. However, these simple models do not provide enough details to emulate actual environments and robot behaviours. The $2 \mathrm{D}$ nature of the simulator is also very limiting if the robots have higher mobility, for example, three dimensional movement. This limitation makes it unsuitable for our research where the robots are being specifically designed to move in a three dimensional space. Gazebo [29] is an open source 3D simulator that can be used with Player. It uses an open source physics engine called Open Dynamics Engine [30] to provide detailed simulation of Newtonian physics of rigid body systems. The three dimensional virtual environment is described in XML format in a world file. Gazebo seems to address the issues with Stage by providing 3D environments and detailed dynamics simulations. However, the most limiting factor of Gazebo is the use of a text based world file for describing the virtual environments. This approach makes it extremely difficult to design and test complex scenarios.

USARSim is based on a commercially available industrial strength game engine called Unreal by Epic Games [31]. The game can be bought at a small 


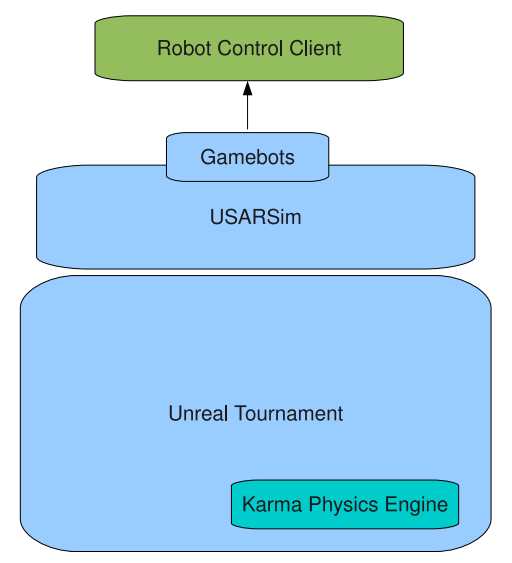

Fig. 8: Structure of USARSim

cost of $£ 20$ to $£ 30$ and the simulator is an open source free software. Unreal gaming environment has an integrated physics engine called Karma [32] that provides high fidelity Newtonian physics simulations for rigid bodies and joint systems. An additional benefit of using the gaming engine is the availability of good quality graphics rendering and visual feedback. By off loading these two important tasks to the gaming engine, USARSim makes it easy for a user to focus on algorithm development for the application. However, the user is still forced to make realistic assumptions due to the high fidelity simulation of the behaviour of robot and its interaction with the surrounding environment. USARSim relies on Gamebots [33] to provide a TCP/IP interface to the outside world. This allows the controller programs to connect to the simulation environment and spawn and control different robots. Fig. (8) shows the overall structure of USARSim simulator and how different components fit together.

Unreal gaming engine also ships with a 3D editor UnrealEd that can be used to easily create $3 \mathrm{D}$ virtual environments. It includes all the necessary facilities to easily create the target environments for our research. The most important of these facilities is the ability to create a Water Volume. A water volume is a region of space where the physics engine modifies its behaviour to simulate a fluid. Thus, in a water volume, a robot experiences fluid friction and buoyancy in addition to gravity. It is also possible to change the default values of these parameters to match those of an actual pond. Using UnrealEd, we have created virtual 3D storage pools. We can also create towers of skips and other clutter of different sizes in these simulated pools. Therefore, this simulation set up can faithfully reproduce the actual storage pools in the virtual simulated environment.

Due to the above mentioned benefits, we decided to use USARSim for investigating the mapping, exploration and localization algorithms for our underwater mobile sensing system. Fig. (9) shows a screen shot of one of the pools in UnrealEd and a robot moving around in this pool in the USARSim simulator. 


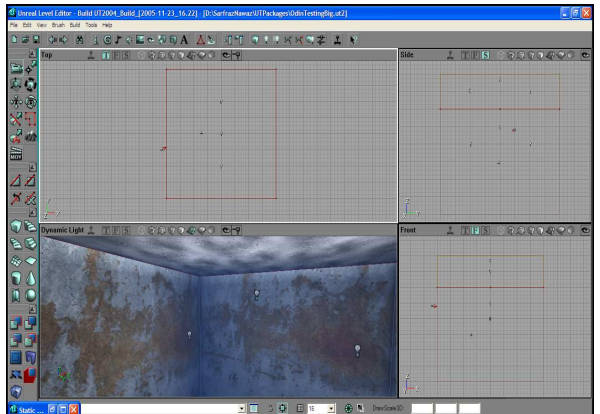

(a) UnrealEd

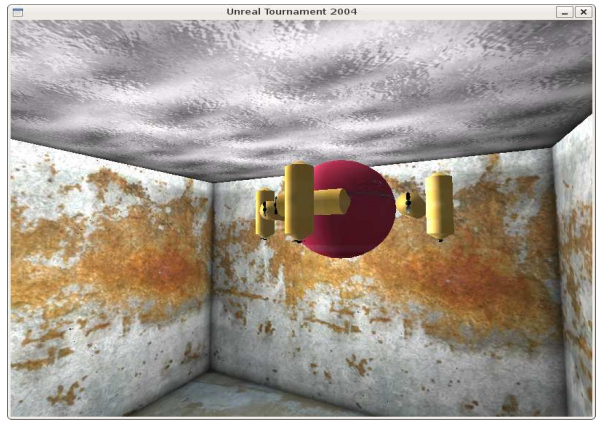

(b) USARSim

Fig. 9: Screenshot of the simulated pond and the robot

\section{Other Industries}

The technologies and the algorithms developed during the course of this research can be used in a wide range of applications in addition to nuclear waste monitoring. In this section, we outline some of the potential industries that operate aqueous environments similar to waste storage ponds and thus can significantly benefit from a networked mobile sensing system for these underwater environments.

Industrial scale process monitoring in chemical industry is an area that can benefit from our contributions. A large portion of these processes involve large scale vessels where different chemicals are mixed together. For these processes, the chemical engineers are generally interested in temperature, pressure, concentration of chemicals, turbidity and other parameters inside the vessel. At the moment, the only technique available to measure some of these parameters is tomography where sensors are placed outside the vessel. However, only some of the above parameters can be estimated using tomography. Another disadvantage of tomography is that it offers very limited spatial and temporal resolution. A swarm of our submersible robots can be placed inside such vessels to perform high density spatial measurements of all of these parameters. These measurements would allow the chemical engineers to develop a better understanding of these chemical processes. This data can then also be used to redesign and improve the chemical processes in terms of energy savings and material usage. The robots can also be used to monitor the wear and tear of reaction vessels from the inside.

Almost all of the chemical plants have large scale settling ponds that are used to clean water after it has been used in a chemical process. These ponds are generally outdoors and could be as big as $100 \mathrm{~m}$ in diameter. These settling ponds are filled with water and the particulates in water are allowed to settle down. The water is then drained off and recycled. Any leakage in these ponds can have a detrimental effect on the surrounding environment. Therefore, it is 
necessary to monitor these ponds for any such leakages. Current methods used for these inspections are manual and thus expensive and time consuming. Our system of networked robots can be used to continuously monitor the pond for any anomalies at significantly reduced costs.

Another industry where our mobile sensor system can be used is sewage wastewater treatment. The wastewater generated by our cities and communities is on average $99.94 \%$ water by weight and only $0.06 \%$ solid waste [34]. Wastewater treatment plants collect this sewage and process it in an extensive network of chambers and ponds to separate these solid wastes from the water. The first stage of this network is similar to settling ponds mentioned above where the water is held for few hours and the solid waste is allowed to settle down. The second stage consists of a large number of aeration ponds where microorganisms are used to breakdown the dissolved waste materials. This process is similar to essentially what happens in nature. However, in a wastewater treatment plant the speed of this process has to increased to process the large quantities of water. Therefore, it is necessary to create optimum conditions for microorganisms in these ponds. This includes mixing the right quantities of oxygen and nutrients in these ponds. Our mobile sensor network can be used in these ponds to monitor the concentration of these substances. Using these observations, the plant engineers can control the amount of oxygen and nutrients injected into the ponds as the wastewater concentration changes every few hours during the course of the entire day.

\section{Related Work}

In this section, we outline some of the related work to put our research in proper perspective. We discuss some underwater autonomous robots and robotic swarm systems and highlight the challenges that differentiate our research from these efforts.

It is generally more difficult to work with underwater environments as compared to the terrestrial one and therefore the development of underwater robots and especially autonomous underwater vehicles (AUVs) has been relatively slow. However, the military, scientific and commercial applications of these autonomous underwater vehicles has contributed to a very rapid progress in recent years. A database of currently available AUVs hosted by Autonomous Undersea Systems Institute lists a total of 103 AUVs available from 51 different manufacturers [35]. More sophisticated systems that consist of swarms of cooperating AUVs, nodes anchored to the seabed and floating buoys have also been developed. Autonomous Ocean Sampling Network [36] and Autonomous Systems Network [37] are a couple of examples of such underwater networked sensing systems. The deployment and coverage analysis of such underwater sensing systems has also been studied [38]. However, almost all of these platforms and networked systems are targeted towards and deployed in open ocean environments. Open sea is an extremely vast environment that presents its own unique challenges. The AUVs used in this environment are relatively large in size and 
have significant movement, sensing and computational resources available on board. Networks of such vehicles are also of relatively large size with individual communication links usually stretching to tens of kilometers. Our application, on the other hand, requires the development of very small scale robots that can move in cluttered aqueous environments with dimensions ranging from tens to a few hundred meters. In addition to being small, these robots must have enough resources to safely navigate, sense and map these constrained cluttered ponds.

Serafina is a small scale AUV developed at Australian National University [39]. It is $50 \mathrm{~cm}$ long and weighs around $5 \mathrm{~kg}$. It has five fixed thrusters with two in horizontal and three in vertical configuration. These thrusters provide five degrees of freedom of movement to the AUV. An inertial sensor and a compass are used to perform attitude control and communication among different nodes is achieved through a long wave radio with a carrier frequency of $122.88 \mathrm{kHz}$. The relative distance and heading of nearby nodes in a swarm of Serafina vehicles is determined by using a pair of hydrophones to receive acoustic signals. However, the performance of these techniques has only been measured in a small tank with clear line of sight and without any clutter [40]. Kalantar and Zimmer [41] have also proposed swarming algorithms to track and map environmental fields like chemical concentration spread and ocean bottom mapping.

Researchers have always looked towards nature to draw inspiration for their own creations and this has resulted in various bio-mimetic robots. The robotic fish [42] developed at University of Essex is one such underwater autonomous robot. It uses the oscillatory movement of its body just like an actual fish to propel itself through water instead of using the traditional propeller based propulsion systems. It can move autonomously in an unknown environment in a complete 3D manner. Researchers at University of Essex are proposing to use these autonomous robots for pollution monitoring in sea ports. The current generation of this robot is $52 \mathrm{~cm}$ long. Three servo motors and mechanical joints are used to generate the oscillatory movement of the body. Four IR sensors and one sonar sensor is used for obstacle detection. However, at the moment there is no communication functionality available to exchange information between different robotic fishes. Therefore, these robots cannot collaborate with each other to form swarms of mobile sensors.

Hydron was developed as part of the Hydra project [43]. It is a very small underwater robot that has a roughly spherical shape with approximately $11 \mathrm{~cm}$ diameter. Movement in the horizontal direction is achieved by expelling water that is drawn in through an impeller from one of the four nozzles selected through a rotating collar. A syringe is used to draw in or expel the water through the bottom. This alters the buoyancy of the robot and thus allows movement in vertical direction. Each hydron robot can communicate with other units with short range optical transceivers. Hydron robots were developed as basic units that could act as building blocks to automatically form more complex structures by rearranging themselves around each other. However, these units are not equipped with localization facilities that could allow them to determine their position in the aqueous environment. 
The cluttered nature of industrial ponds creates a very harsh environment for communication and positioning systems and the focus of our research is to address this challenge at each individual layer of our mobile sensing system ranging from low level distance estimation to application level mapping and exploration algorithms. This is a unique application scenario and to the best of our knowledge has never been addressed before. The robotic platforms and sensing systems that we discussed in this section are either too large for our purposes or have never been tested in our unique application scenario.

\section{Conclusion}

In this paper, we discussed a novel application of nuclear waste storage pool monitoring and outlined a detailed architecture of our proposed underwater mobile sensor network for monitoring these pools. We described the difficulties faced by robot localization and environment mapping algorithms due to the cluttered and enclosed nature of these underwater environments with preliminary results. We outlined the future research challenges of our unique application and also highlighted various other industrial applications that can potentially benefit from our underwater mobile sensor network.

Acknowledgements This work is supported by the EPSRC grants EP/F064209/1 and EP/F064578/1 on Actuated Acoustic Sensor Networks for Industrial Processes (AASN4IP). We would also like to thank the National Nuclear Laboratory (NNL) for their contribution to this paper.

\section{References}

1. World Nuclear Association. http://www. world-nuclear.org.

2. L E J Roberts. Radioactive waste management. Annual Review of Nuclear and Particle Science, 40(1):79-112, 1990.

3. Charles D. Hollister and Steven Nadis. Burial of radioactive waste under the seabed. Scientific American, January 1998.

4. Thor I. Fossen. Guidance and Control of Ocean Vehicles. John Wiley \& Sons, 1994.

5. M. Krieg and K. Mohseni. Thrust characterization of a bioinspired vortex ring thruster for locomotion of underwater robots. Oceanic Engineering, IEEE Journal of, 33(2):123-132, April 2008.

6. K. Whitehouse, C. Karlof, A. Woo, F. Jiang, and D. Culler. The effects of ranging noise on multihop localization: an empirical study. In Information Processing in Sensor Networks, 2005. IPSN 2005. Fourth International Symposium on, pages 73-80, 2005.

7. A. Savvides, C.C. Han, and M.B. Strivastava. Dynamic fine-grained localization in ad-hoc networks of sensors. In Proceedings of the 7th annual international conference on Mobile computing and networking, pages 166-179. ACM New York, NY, USA, 2001. 
8. J. Liu, Y. Zhang, and F. Zhao. Robust distributed node localization with error management. In Proceedings of the 7th ACM international symposium on Mobile ad hoc networking and computing, pages 250-261. ACM New York, NY, USA, 2006.

9. Z. Yang and Y. Liu. Quality of Trilateration: Confidence Based Iterative Localization. In Distributed Computing Systems, 2008. ICDCS'08. The 28th International Conference on, pages 446-453, 2008.

10. Vijay Chandrasekhar, Winston KG Seah, Yoo Sang Choo, and How Voon Ee. Localization in underwater sensor networks: survey and challenges. In WUWNet '06: Proceedings of the 1st ACM international workshop on Underwater networks, pages 33-40, New York, NY, USA, 2006. ACM.

11. M. Erol, L. Vieira, A. Caruso, F. Paparella, M. Gerla, and S. Oktug. Multi stage underwater sensor localization using mobile beacons. In Sensor Technologies and Applications, 2008. SENSORCOMM '08. Second International Conference on, pages 710-714, August 2008.

12. M. Erol, L.F.M. Vieira, and M. Gerla. Auv-aided localization for underwater sensor networks. In Wireless Algorithms, Systems and Applications, 200\%. WASA $200 \%$. International Conference on, pages 44-54, Aug. 2007.

13. Enhanced Wireless 911 Services. http://www.fcc.gov/Bureaus/Wireless/News_ Releases/1999/nrw19040.html.

14. M.P. Wylie and J. Holtzman. The non-line of sight problem in mobile location estimation. Universal Personal Communications, 1996. Record., 1996 5th IEEE International Conference on, 2:827-831 vol.2, Sep-2 Oct 1996.

15. L. Cong and Weihua Zhuang. Non-line-of-sight error mitigation in tdoa mobile location. Global Telecommunications Conference, 2001. GLOBECOM '01. IEEE, 1:680-684 vol.1, 2001.

16. P.C. Chen. A non-line-of-sight error mitigation algorithm in locationestimation. In Wireless Communications and Networking Conference, 1999. WCNC. 1999 IEEE, pages 316-320, 1999.

17. S. Venkatraman, J. Caffery Jr, and H.R. You. Location using LOS range estimation in NLOS environments. In Vehicular Technology Conference, 2002. VTC Spring 2002. IEEE 55th, volume 2, 2002.

18. J. Borras, P. Hatrack, and N.B. Mandayam. Decision theoretic framework for nlos identification. Vehicular Technology Conference, 1998. VTC 98. 48th IEEE, 2:1583-1587 vol.2, May 1998.

19. S. Al-Jazzar and J. Caffery Jr. New algorithms for nlos identification. In Proceedings of the 14th IST Mobile and Wireless Communications Summit, 2005.

20. S. Al-Jazzar, J. Caffery Jr, and H.R. You. A scattering model based approach to NLOS mitigation in TOA location systems. In Vehicular Technology Conference, 2002. VTC Spring 2002. IEEE 55th, volume 2, 2002.

21. Yiu-Tong Chan, Wing-Yue Tsui, Hing-Cheung So, and Pak chung Ching. Timeof-arrival based localization under nlos conditions. Vehicular Technology, IEEE Transactions on, 55(1):17-24, Jan. 2006.

22. Randall C. Smith and Peter Cheeseman. On the Representation and Estimation of Spatial Uncertainty. The International Journal of Robotics Research, 5(4):56-68, 1986.

23. Sebastian Thrun. Robotic mapping: a survey. pages 1-35, 2003.

24. A. Elfes. Sonar-based real-world mapping and navigation. IEEE Journal of Robotics and Automation, 3(3):249-265, June 1987.

25. Hans Moravec. Sensor fusion in certainty grids for mobile robots. AI Magazine, 9(2):61-74, 1988. 
26. Hans Moravec. Robot spatial perception by stereoscopic vision and 3d evidence grids. Technical Report CMU-RI-TR-96-34, Robotics Institute, Pittsburgh, PA, September 1996.

27. B. Yamauchi. A frontier-based approach for autonomous exploration. pages 146 151, Jul 1997.

28. B. Gerkey, R. Vaughan, and A. Howard. The Player/Stage Project: tools for multi-robot and distributed sensor systems. In 11th International Conference on Advanced Robotics (ICAR 2003), Coimbra, Portugal, June 2003.

29. Gazebo. http://playerstage. sourceforge.net/index.php?src=gazebo.

30. Russell Smith. Open dynamics engine. http://www.ode.org.

31. Epic Games. http://www.epicgames.com.

32. Karma physical engine. http://wiki.beyondunreal.com/wiki/Karma.

33. Gal A. Kaminka, Manuela M. Veloso, Steve Schaffer, Chris Sollitto, Rogelio Adobbati, Andrew N. Marshall, Andrew Scholer, and Sheila Tejada. Gamebots: a flexible test bed for multiagent team research. Commun. ACM, 45(1):43-45, 2002.

34. Following the flow: An inside look at wastewater treatment. Online, http://www.wef.org/AboutWater/ForThePublic/WastewaterTreatment.

35. AUV Database. http://auvac.org/resources/browse/.

36. Autonomous Ocean Sampling Network (AOSN) II. http://www.princeton.edu/ $\sim$ dcsl/aosn.

37. Steven G. Chappell, Rick J. Komerska, D. Richard Blidberg, Christiane N. Duarte, Gerald R. Martel, Denise M. Crimmins, Michel A. Beliard, Robert Nitzel, James C. Jalbert, and Radim Bartos. Recent field experiences with multiple cooperating solar-powered vehicles. In Fifteenth International Symposium on Unmanned Untethered Submersible Technology, Durham, NH, August 2007.

38. Dario Pompili, Tommaso Melodia, and Ian F. Akyildiz. Three-dimensional and two-dimensional deployment analysis for underwater acoustic sensor networks. Ad Hoc Networks, 7(4):778-790, 2009.

39. Felix Schill. Distributed Communication in Swarms of Autonomous Underwater Vehicles. PhD thesis, The Australian National University, July 2007.

40. Navinda Kottege and Uwe R. Zimmer. Acoustical methods for azimuth, range and heading estimation in underwater swarms. In Acoustics '08, Palais des Congrs, Paris, June 2008.

41. S. Kalantar and U.R. Zimmer. Scale-adaptive polygonal formations of submersible vehicles and tracking isocontours. pages 3146-3151, September 2008.

42. Huosheng $\mathrm{Hu}$. Biologically inspired design of autonomous robotic fish at Essex. In IEEE SMC Chapter Conference on Advances in Cybernetic Systems, Sheffield, UK, September 2006.

43. Esben Hallundbk stergaard, David J. Christensen, Peter Eggenberger, Tim Taylor, Peter Ottery, and Henrik Hautop Lund. Hydra: From cellular biology to shape-changing artefacts. In Wlodzislaw Duch, Janusz Kacprzyk, Erkki Oja, and Slawomir Zadrozny, editors, Artificial Neural Networks: Biological Inspirations ICANN, volume 3696 of Lecture Notes in Computer Science, pages 275-281. Springer, 2005 